\title{
Unconventional Vortex Dynamics in Mesoscopic Superconducting Corbino Disks
}

\author{
N. S. Lin, V. R. Misko, and F. M. Peeters \\ Department of Physics, University of Antwerpen, Groenenborgerlaan 171, B-2020 Antwerpen, Belgium
}

(Received 6 March 2009; published 14 May 2009)

\begin{abstract}
The discrete shell structure of vortex matter strongly influences the flux dynamics in mesoscopic superconducting Corbino disks. While the dynamical behavior is well understood in large and in very small disks, in the intermediate-size regime it occurs to be much more complex and unusual, due to (in) commensurability between the vortex shells. We demonstrate unconventional vortex dynamics (inversion of shell velocities with respect to the gradient driving force) and angular melting (propagating from the boundary where the shear stress is minimum, towards the center) in mesoscopic Corbino disks.
\end{abstract}

PACS numbers: 74.25.Qt, 74.78.Na

Investigation of mesoscopic and nanostructured superconductors has become one of the major topics of experimental and theoretical studies on vortex matter in superconductors over the past two decades. The unique possibility to investigate the interplay between the vortexvortex and vortex-boundary interactions, the size-, shape-, and symmetry-induced effects makes mesoscopic superconductors very attractive from both the fundamental research and possible fluxonics applications, i.e., manipulating single flux quanta similar to electrons in nanoand microelectronics. Thus it was demonstrated theoretically [1-4] and experimentally [5-8] that in mesoscopic superconducting disks shape effect leads to the formation of circular-symmetric giant vortex states $[5,6]$ or concentric shells of vortices [7,9]. Furthermore, symmetryinduced vortex-antivortex "molecules" were theoretically predicted [10] in mesoscopic squares and triangles. On the other hand, the dynamics of vortex matter in disks can be studied using a Corbino setup. In the Corbino geometry an applied current produces a gradient in the Lorentz force, i.e., introduces a shear driving force between the rings (in large disks [11]) or shells (in mesoscopic regime [12]) of vortices. This provides the unique opportunity to study various dynamical effects related to vortex motion, e.g., the onset of plasticity, channeling [13], vortex friction [14], etc. Moreover, the dynamics of self-organized vortex matter in mesoscopic disks has many common features to, e.g., atomic matter, charged particles in Coulomb crystals, vortices in rotating Bose-Einstein condensates, magnetic colloids, synthetic nanocrystals, etc. [15-17], or charged balls diffusing in macroscopic Wigner rings [18].

Several early studies on vortex matter in Corbino disks focused on the transition from elastic to plastic vortex motion $[11,19,20]$. Indeed, the radial current density $j$ in a Corbino disk decays as $I_{0} / r$ along the radius resulting in a stronger Lorentz force $F_{L}$ driving vortices near the disk center than near the edge. For small driving currents, the local shear stress is small and the whole vortex pattern, although elastically deformed, moves as a rigid body. For applied currents larger than some critical value $I_{c}$ a strong spatially inhomogeneous stress breaks up the vortex solid, and concentric annular regions (in large disks) or shells (in mesoscopic disks) move with different angular velocities. The different dynamical phases (e.g., elastic motion, shearinduced plastic slip) of vortex motion can be detected by measuring voltage profiles [11]. In large Corbino disks, the onset of plasticity was theoretically analyzed within a continuous model [20] and using molecular dynamics (MD) simulations of interacting vortices at $T=0$ [21]. The magnetization and different scenarios of magneticflux penetration in a thin Corbino disk were studied [22] using the critical-state model. The dynamics of vortex shells in small mesoscopic Corbino disks containing up to 40 vortices (cf. Ref. [7]) arranged in two- and three-shell configurations, was studied using MD simulations for $T=$ 0 and $T>0$ [12]. It was shown that the critical current $I_{c}$ had a nonmonotonic dependence on the magnetic field $h$. The dynamical instabilities associated with a jump in $I_{c}(h)$ were revealed. The found unusual behavior was shown to be related to a "structural transition," i.e., an intershell vortex transition with increasing $h$. In the presence of pinning, thermally activated externally driven flux motion was analyzed for $T>0$. The investigation of the angular thermal melting explained the experimentally observed two-step melting transition in Corbino disks [11].

Here, we study the dynamics of vortex shells in larger mesoscopic Corbino disks containing several shells. The essential qualitative difference to the earlier studied case [12] is the following: While in small disks vortex shells are nearly perfectly circular, due to the strong confinement, in larger disks the vortex-vortex interaction becomes more important. The dynamics of vortex shells is influenced, to a great extent, by the interplay between the gradient Lorentz force and the (in)commensurability effects between the numbers of vortices in adjacent (and even more remote) vortex shells. Note that all of these factors are sensitive to the radius of the disk. As a result of the complex interplay of the above five factors, i.e., (a) confinement, (b) the intervortex interaction, (c) the gradient Lorentz force, (d) (in)commensurability, and (e) the size of the disk, the system of vortex shells in large Corbino disks demonstrates very rich and unusual dynamical behavior. Thus we found 
(i) unconventional angular melting, i.e., when the melting first occurs in regions where the shear stress is minimum, and (ii) unconventional shell dynamics when a shell that experiences a weaker Lorentz force moves faster than the adjacent shell driven by a stronger force. We expect that our results can be useful for understanding and predicting the dynamics of complex systems of interacting particles confined to an external potential such as colloids, vortices in Bose-Einstein condensates, charged particles, etc., or even for better understanding of mechanisms of motion of some biological objects.

Theory and simulation.-We place a Corbino disk which has thickness $d$ and radius $R$ in a perpendicular external magnetic field $\boldsymbol{H}_{0}$. An external current flows radially from the center to the edge of the disk and results in the inhomogeneous sheath current density $j(\rho)=I_{\text {ext }} / 2 \pi \rho d$. The Lorentz force (per unit length) acting on vortex $i$, $\Phi_{0} \boldsymbol{j} \times \hat{z}$, resulting from the external current is

$$
\boldsymbol{f}_{i}^{d}=\frac{\Phi_{0} I_{\mathrm{ext}}}{2 \pi d} \frac{\boldsymbol{\rho}_{i} \times \hat{z}}{\rho_{i}^{2}}=f_{0} I_{0} \frac{\boldsymbol{r}_{i} \times \hat{z}}{r_{i}^{2}},
$$

where $\boldsymbol{\rho}_{i}$ is the vortex position, $\boldsymbol{r}_{i}=\boldsymbol{\rho}_{i} / R$, and $\hat{z}$ is the unit vector along the magnetic field direction. Here the unit of force $f_{0}=\Phi_{0}^{2} / 2 \pi \mu_{0} R \lambda^{2}=4 \pi \mu_{0} \xi^{2} H_{c}^{2} / R$ and $I_{0}=$ $\mu_{0} \Lambda I_{\text {ext }} / \Phi_{0}$ are expressed in terms of the coherence length $\xi$, the magnetic field penetration depth $\lambda\left(\Lambda=\lambda^{2} / d\right.$ is the effective penetration depth in a superconductor of thickness $d$ ), and the thermodynamic $H_{c}$ and the upper $H_{c 2}$ critical magnetic fields. In a thin superconducting disk such that $d<\xi \ll R \ll \Lambda$, the vortex-vortex interaction force $f_{i}^{\mathrm{vV}}$ and the force of the vortex interaction with the shielding currents and with the edge $\boldsymbol{f}_{i}^{s}$ can be modeled, respectively, by [23-25]

$$
\begin{gathered}
\boldsymbol{f}_{i}^{\mathrm{vv}}=f_{0} \sum_{i, k}^{L}\left(\frac{\boldsymbol{r}_{i}-\boldsymbol{r}_{k}}{\left|\boldsymbol{r}_{i}-\boldsymbol{r}_{k}\right|^{2}}-r_{k}^{2} \frac{r_{k}^{2} \boldsymbol{r}_{i}-\boldsymbol{r}_{k}}{\left|r_{k}^{2} \boldsymbol{r}_{i}-\boldsymbol{r}_{k}\right|^{2}}\right), \\
\boldsymbol{f}_{i}^{s}=f_{0}\left(\frac{1}{1-r_{i}^{2}}-h\right) \boldsymbol{r}_{i},
\end{gathered}
$$

where $h=\left(H_{0} / 2 H_{c 2}\right)(R / \xi)^{2}$ is the dimensionless applied magnetic field, and $L$ is the number of vortices, or the vorticity. Our numerical approach is based on the Langevin dynamics algorithm, where the time integration of the equations of motion is performed in the presence of a random thermal force. The overdamped equation of motion (see, e.g., [26,27]) becomes $\eta \boldsymbol{v}_{i}=\boldsymbol{f}_{i}=\boldsymbol{f}_{i}^{\mathrm{Vv}}+\boldsymbol{f}_{i}^{\mathrm{vp}}+$ $\boldsymbol{f}_{i}^{T}+\boldsymbol{f}_{i}^{d}+\boldsymbol{f}_{i}^{s}$. Here $\boldsymbol{f}_{i}^{d}$ is the driving force [Eq. (1)], $\boldsymbol{f}_{i}^{\mathrm{vp}}$ is the force due to vortex-pin interaction, and $\boldsymbol{f}_{i}^{T}$ is the thermal stochastic force, obeying the condition $\left\langle\boldsymbol{f}_{\alpha, i}(t) \boldsymbol{f}_{\beta, j}\left(t^{\prime}\right)\right\rangle=2 \eta \delta_{\alpha \beta} \delta_{i j} \delta\left(t-t^{\prime}\right) k_{B} T$, where Greek and italic indices refer to vector components and vortex labels, $\eta$ is the viscosity which is set to unity. The ground state of the system is obtained by minimization of the London free energy, taking into account the vortex cores contribution [28], with respect to (i) different $L$, and (ii) different vortex configurations for the same $L$ (see Refs. [24,25]) by performing the stimulated annealing simulation (SAS) that simulates field-cooling experiments.

Angular melting: commensurability vs the Lorentz force gradient.-In a Corbino disk the gradient of the Lorentz force is maximum near the center, and thus one can expect that with increasing applied current the growing shear stress first breaks the vortex solid near the center and then, with further increasing the applied current, the angular melting process $[29,30]$ propagates from the center to the peripheral regions [31]. Indeed, this scenario of angular melting of the vortex solid in Corbino disks was observed in the experiment [11] and analyzed theoretically (e.g., [19-21]). Similarly, in mesoscopic disks vortex shells first unlock near the center with increasing either applied current or temperature [12]. Figure 1 shows the angular velocity of different vortex shells $\omega_{i}$ for the configuration (1, $5,12,18)$ as a function of the applied current $I_{0}$. At low $I_{0}$, the whole vortex configuration rotates as a rigid body while at some critical value $I_{c 12}$ the first inner shell splits off and starts to rotate separately with a higher angular velocity $\omega_{1}$. At a higher driving current $I_{c 23}$, the Lorentz force gradient becomes sufficient for unlocking the second shell. For vortex configuration with a larger number of shells, the angular melting thus occurs gradually through the consequent unlocking of the shells from the center towards the periphery.

It is easy to understand that the above "classical" behavior is the only possible scenario for angular melting of the vortex solid in large (macroscopic) Corbino disks.

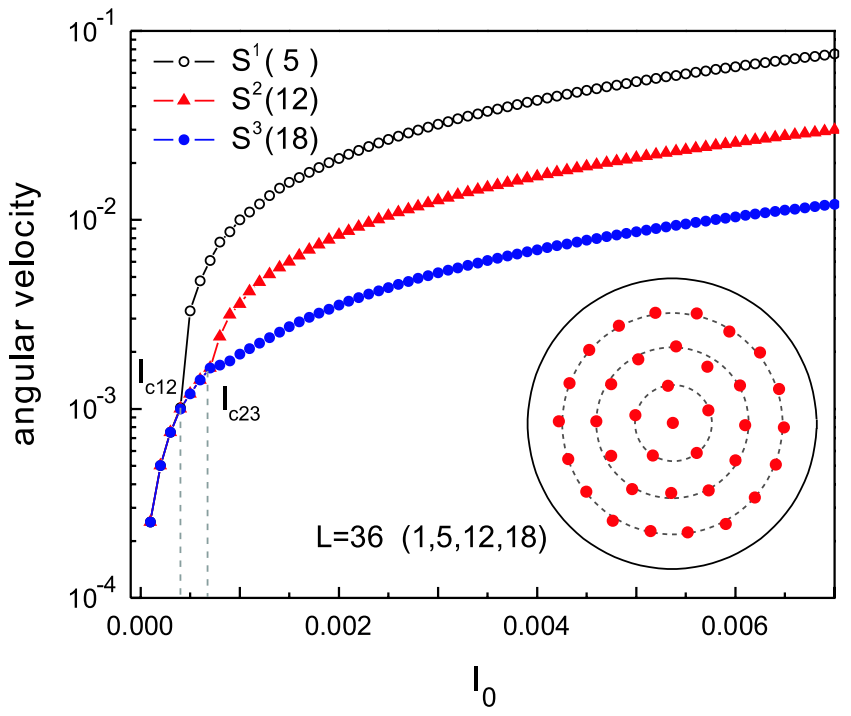

FIG. 1 (color online). The angular velocities $\omega_{i}$ of different vortex shells as a function of the external driving current $I_{0}$. The critical values $I_{c 12}$ and $I_{c 23}$ correspond to the unlock transition between the shells $S^{1}-S^{2}$ and $S^{2}-S^{3}$. The two-step angular melting starts at the center of the disk where the Lorentz force is maximum. Inset: the shell configuration $(1,5,12,18)$ for the vorticity $L=36$ and $h=45$. The dashed gray circles showing different shells are guides for eye. 
Indeed, in large disks (i.e., where the intervortex distance $a \ll R$ ) vortices form a uniform hexagonal Abrikosov lattice. It is clear that the shear modulus of such lattice is the same everywhere (except for the regions in the vicinity to the boundary). Thus, the strongest shear stress which occurs near the center of the disk breaks up the lattice first. However, in the case of vortex shells in mesoscopic disks the situation turns out to be very different: the shear modulus becomes a function of the radius due to (in) commensurate number of vortices in different adjacent shells. (Moreover, the shear modulus can also depend on the angle if the circular symmetry of the vortex-shell configuration is broken by defects.) Thus we can expect that the angular melting in a system of vortex shells will happen in a different way if the shear modulus has a strong radial dependence, i.e., stronger than the difference in Lorentz force between adjacent shells.

Because of the complexity of the system it is not possible to find the shear modulus analytically. However, we know that the shear modulus will be much smaller for incommensurate shells than for the commensurate case where "magic number configurations" [29,32] make the vortex system more rigid. If the inner shells are incommensurate, the Lorentz force gradient will facilitate the classical scenario of angular melting of the vortex solid and the unlocking of shells will occur at an even lower value of the driving current $I_{0}$. In contrast, if the outer shells are incommensurate, the friction between them can be so small that they first unlock, leading to unconventional angular melting, i.e., from the outer edge of the vortex solid inwards.

We analyzed different ground-state and metastable vortex-shell configurations for different number of vortices $L$ and found different scenarios of angular melting in a mesoscopic Corbino disk. The most unusual way of melting is when it starts from the outermost shell and propagates towards the center of the disk, i.e., opposite to the classical way. An example of such unconventional melting is presented in Fig. 2. Note that it is realized for the groundstate energy configuration $(1,6,12,19)$ for $L=38$. The circularlike incommensurate shell with 19 vortices easily slides with respect to the adjacent shell with 12 vortices even for very low driving currents $I>I_{c 23}$ while the hard "core" $(1,6,12)$ can sustain much stronger shear stress and the inner shell becomes unlocked at $I_{c 12} \gg I_{c 23}$ (Fig. 2).

Unconventional dynamics of vortex shells.-The interplay between (in)commensurability effects of vortex shells, on the one hand, and the inhomogeneous Lorentz force, on the other hand, can lead to even more striking and unexpected behavior. Thus, we demonstrate here that these effects can result in a very unusual ("unconventional") dynamics of vortex shells in a mesoscopic Corbino disk. In particular, we found that in the region of the multistep transition from a "rigid body" rotation to individual rotation of vortex shells, an inversion of the angular velocities with respect to the gradient of the Lorentz force can occur.

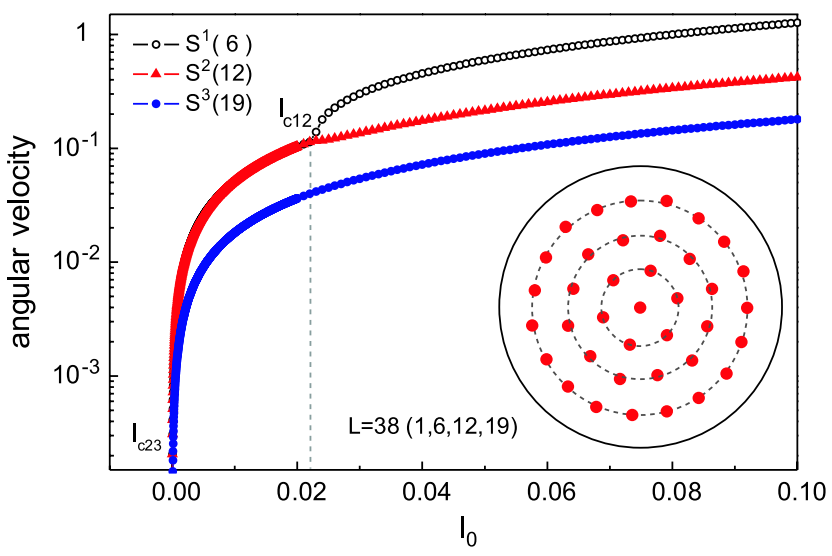

FIG. 2 (color online). Unconventional angular melting of vortex shells for the configuration $(1,6,12,19)$ : the two-step melting starts near the boundary where the Lorentz force is minimum. The inset shows the shell configuration $(1,6,12$, 19) for the vorticity $L=38$ and $h=50$.

For unlocked shells we expect that, due to the $1 / r$ dependence of the driving force, the angular velocity of the different shells is a monotonically decreasing function of the radius. This is clearly seen in Figs. 1 and 2. Very surprisingly, we found situations were this rule is broken as demonstrated in Fig. 3 for the $L=93$ configuration (1, $6,12,19,25,30)$. The incommensurate shell $S^{4}$ with 25 vortices rotates faster than the adjacent shell $S^{3}$ (which is closer to the center than $S^{4}$ ) for $I_{c 234} \approx 0.004 \lesssim I_{0} \lesssim$ $I_{c 34-43} \approx 0.0095$. This puzzling behavior is a consequence of the interplay between the Lorentz force, (in)commensurability effects, and shell defects. For the above range of $I_{0}$, the vortex-shell configuration is only partially split. The strong commensurate inner-shell core rotates as a rigid body while incommensurate outer shells seem to rotate separately. However, their motion turns out to be correlated in a rather complex manner. Namely, the $S^{3}$ shell with 19 vortices has a "defect," i.e., one extra vortex (indicated by a blue or dark gray circle in the inset of Fig. 3) which makes this shell incommensurate with the hexagonlike core $(1,6,12)$. However, due to the intershell vortex-vortex interaction a part of this shell squeezes and adjusts itself to the core, thus making the other part of the shell (i.e., incommensurate, containing the defect) stretched. The commensurate part (CP) of the $S^{3}$ shell (the hexagonlike part of shell $S^{3}$-see inset of Fig. 3) is locked to the core while the incommensurate part (ICP) (the "circular" part of shell $S^{3}$-see inset of Fig. 3) is unlocked and slides with respect to the core. Thus the motion of the $S^{3}$ shell with respect to the core occurs in the form of a compressiondecompression wave propagating in the direction opposite to the direction of the rotation. This backward wave motion reduces the average angular velocity of the $S^{3}$ shell by a factor of $18 n / 19$ where $n=2 \pi r_{S 3} / \lambda_{\mathrm{CP}-\mathrm{ICP}}$ and $\lambda_{\mathrm{CP}-\mathrm{ICP}}$ is the wavelength of the CP-ICP compression-decompression wave [33]. At the same time, the incommensurate $S^{4}$ shell with 25 vortices, due to the friction with the $\mathrm{CP}$ of the 


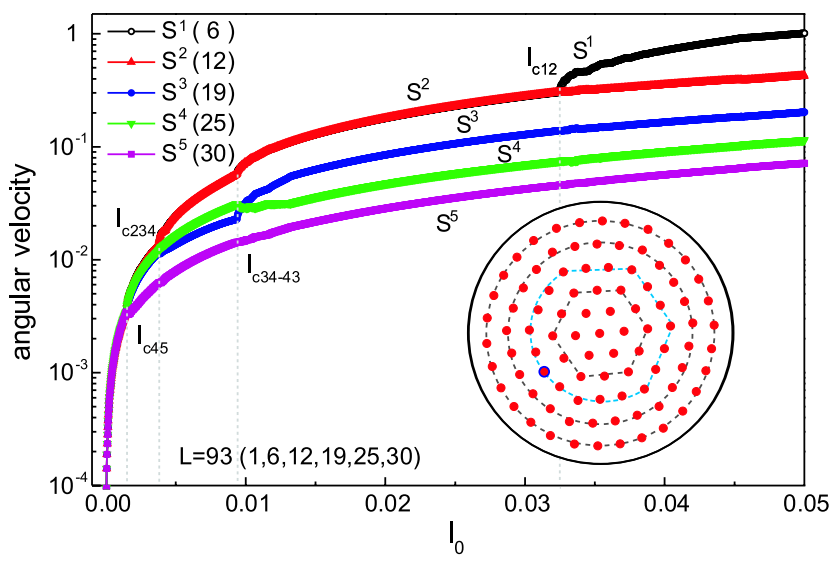

FIG. 3 (color online). $\quad \omega_{i}\left(I_{0}\right)$ for the configuration $(1,6,12,19$, $25,30)$. The system displays unconventional vortex dynamics in the region $I_{c 234} \approx 0.004 \lesssim I_{0} \lesssim I_{c 34-43} \approx 0.0095$ where the shell $S^{4}$ (with 25 vortices), that experiences a weaker Lorentz force, rotates faster than the adjacent shell $S^{3}$ (19 vortices) driven by a larger Lorentz force. The inset shows the shell configuration $(1,6,12,19,25,30)$ for the vorticity $L=93$ and $h=109$. The $S^{3}$ shell consists of a hexagonlike part (CP) commensurate with the hexagonal core $(1,6,12)$ and a "circularlike" part (ICP) (with a defect) incommensurate with the core.

$S^{3}$ shell, is accelerated by the faster rotation of the core. Note that this counterintuitive behavior is possible only when a very delicate balance between the vortex-vortex interaction and the Lorentz force is established. When the latter becomes large enough (i.e., for $I_{0} \geq 0.01$ ) the usual vortex-shell velocity distribution (i.e., monotonic decreasing) is recovered (see Fig. 3). Note that at $I_{0} \approx 0.01$ the angular velocity of the core also has a feature (kink) indicating the complete unlocking between the shells $S^{3}$, $S^{4}$ and the core (which itself breaks apart only for very large values of driving, $I_{0}>I_{c 12} \approx 0.0325$ ).

Conclusions. - We found that the interplay between the (in)commensurability effects, the vortex-vortex interaction, and the gradient Lorentz force in a mesoscopic Corbino disk can result in a very unusual and counterintuitive dynamics of vortex shells. Thus we demonstrated the following: (i) unconventional angular melting when, with increasing driving current, the melting first occurs near the boundary where the shear stress is minimum and propagates towards the center, in an opposite way to the classical scenario of conventional angular melting in a Corbino disk; (ii) unconventional shell dynamics when a shell that experiences a weaker Lorentz force moves faster than the adjacent shell driven by a stronger Lorentz force. These examples of strongly nonlinear dynamical behavior can be useful for understanding and prediction of dynamics of other complex interacting systems.

We thank Franco Nori and Fabio Marchesoni for useful discussions. This work was supported by the "Odysseus" program of the Flemish Government, FWO-Vl, and IAP.
V.R. M. is funded by the EU Marie Curie project, Contract No. MIF1-CT-2006-040816.

[1] P. S. Deo et al., Phys. Rev. Lett. 79, 4653 (1997).

[2] V. A. Schweigert and F. M. Peeters, Phys. Rev. B 57, 13817 (1998).

[3] V. A. Schweigert and F. M. Peeters, Phys. Rev. Lett. 83, 2409 (1999).

[4] B. J. Baelus et al., Phys. Rev. B 63, 144517 (2001).

[5] A. K. Geim et al., Nature (London) 390, 259 (1997).

[6] A. Kanda et al., Phys. Rev. Lett. 93, 257002 (2004).

[7] I. V. Grigorieva et al., Phys. Rev. Lett. 96, 077005 (2006).

[8] I. V. Grigorieva et al., Phys. Rev. Lett. 99, 147003 (2007).

[9] V. R. Misko et al., Phys. Rev. B 76, 024516 (2007).

[10] L. F. Chibotaru et al., Nature (London) 408, 833 (2000); Phys. Rev. Lett. 86, 1323 (2001); V. R. Misko et al., Phys. Rev. Lett. 90, 147003 (2003).

[11] G.W. Crabtree et al., J. Low Temp. Phys. 117, 1313 (1999); D. Lopez et al., Phys. Rev. Lett. 82, 1277 (1999).

[12] V.R. Misko and F. M. Peeters, Phys. Rev. B 74, 174507 (2006).

[13] R. Besseling et al., New J. Phys. 7, 71 (2005).

[14] A. Maeda et al., Phys. Rev. Lett. 94, 077001 (2005).

[15] T. B. Mitchell et al., Phys. Rev. Lett. 87, 183001 (2001).

[16] A. Pertsinidis and X.Z. Ling, Nature (London) 413, 147 (2001).

[17] C. Murray et al., Annu. Rev. Mater. Sci. 30, 545 (2000).

[18] G. Coupier et al., Phys. Rev. E 73, 031112 (2006).

[19] Y. Paltiel et al., Phys. Rev. Lett. 85, 3712 (2000).

[20] P. Benetatos and M. C. Marchetti, Phys. Rev. B 65, 134517 (2002).

[21] M.-C. Miguel and S. Zapperi, Nature Mater. 2, 477 (2003).

[22] A. A. Babaei Brojeny and J. R. Clem, Phys. Rev. B 64, 184507 (2001).

[23] A. I. Buzdin and J.P. Brison, Phys. Lett. A 196, 267 (1994).

[24] L. R. E. Cabral et al., Phys. Rev. B 70, 144523 (2004).

[25] B. J. Baelus et al., Phys. Rev. B 69, 064506 (2004).

[26] F. Nori, Science 271, 1373 (1996).

[27] C. Reichhardt et al., Phys. Rev. Lett. 78, 2648 (1997); Phys. Rev. B 57, 7937 (1998); 58, 6534 (1998).

[28] L. R. E. Cabral and F. M. Peeters, Phys. Rev. B 70, 214522 (2004).

[29] V. M. Bedanov and F. M. Peeters, Phys. Rev. B 49, 2667 (1994).

[30] M. F. Laguna et al., Phys. Rev. B 64, 104505 (2001).

[31] This "angular melting," or consequent unlocking of vortex shells, can be induced either by temperature or by shear (i.e., shear-induced plastic deformation). In the latter case, it is similar to the "shear-induced melting transition" in colloidal crystals.

[32] V. A. Schweigert and F. M. Peeters, Phys. Rev. B 51, 7700 (1995).

[33] Note that this kind of motion resembles the motion of a caterpillar whose part of the body "locks" to the surface while the other part moves forward (decompression), and then it locks and pulls the other part (compression). 